\title{
Post-Operative Mortality after Ileal Perforation at a Teaching Hospital in Dhaka City
}

\author{
Mahbuba Begum1 ${ }^{*}$, Mohammad A. Majid², Din Mohammad³, Wahida Begum4, \\ Md. Abdullah Yusuf ${ }^{5}$, Shamima Sultana ${ }^{6}$
}

${ }^{1}$ Department of Surgery, Medical College for Women \& Hospital, Dhaka, Bangladesh

${ }^{2}$ Department of Surgery, Dhaka Medical College, Dhaka, Bangladesh

${ }^{3}$ Department of Surgery, Patuakhali Medical College, Patuakhali, Bangladesh

${ }^{4}$ Department of Neuroradiology \& Imaging, National Institute of Neurosciences \& Hospital, Dhaka, Bangladesh

${ }^{5}$ Department of Microbiology, National Institute of Neurosciences \& Hospital, Dhaka, Bangladesh

${ }^{6}$ National Institute of Ophthalmology \& Hospital, Dhaka, Bangladesh

Email: *mahbuba44k@gmail.com

How to cite this paper: Begum, M., Majid, M.A., Mohammad, D., Begum, W., Yusuf, Md.A. and Sultana, S. (2020) Post-Operative Mortality after Ileal Perforation at a Teaching Hospital in Dhaka City. Surgical Science, $11,25-31$

https://doi.org/10.4236/ss.2020.112004

Received: September 21, 2019

Accepted: February 18, 2020

Published: February 21, 2020

Copyright $\odot 2020$ by author(s) and Scientific Research Publishing Inc. This work is licensed under the Creative Commons Attribution International License (CC BY 4.0).

http://creativecommons.org/licenses/by/4.0/

\begin{abstract}
Background: Ileal perforation is a very critical condition. Objectives: The purpose of the present study was to see the post-operative mortality after ileal perforation. Methodology: This prospective cohort study was conducted in the Department of Surgery at Dhaka Medical College Hospital, Dhaka, Bangladesh from September 2000 to December 2002 for period of 2 years and 3 months. All the patients presented with ileal perforation at any age with both sexes were included in this study. Patients were selected consecutively and the patients who showed ileal perforation at laparotomy were included in this study. Preoperative diagnosis was based on detailed history, complete physical examinations supported by plain x-ray abdomen in erect posture including both domes of diaphragm. After immediate resuscitation surgical treatment was undertaken as soon as possible following admission in all cases. The patients were followed up and the mortality profiles were recorded after surgical intervention. Result: Out of 53 patients having postoperative complications 38 patients were survived and 15 patients were expired. So total survivors were $85 \%$ and non-survivors $15 \%$. In this study most of the mortality (12\%) was attributed to septicemia and mode of death was multiple organ failure. Respiratory complications caused $2 \%$ mortality one patient died of ARDS and another elderly patient with preexisting bronchial asthma developed respiratory failure and was unresponsive to treatment. One death was related to faecal fistula followed by severe fluid, electrolyte and acid-base imbalance with peritonitis and gross sepsis. Conclusion: In conclusion, the mortality is significantly high due to septicemia, ARDS and faecal fistula.
\end{abstract}




\section{Keywords}

Post-Operative, Mortality, Ileal Perforation, Faecal Fistula

\section{Introduction}

The history, clinical examinations and a plain X-ray of the abdomen in erect posture are the main tools for making a diagnosis of perforated ileum [1]. Serological and bacteriological investigations are only supportive to elicit the cause of perforation; however, this doesn't play any role in the emergency management of the patients [2]; these investigations are not done in every case due to lack of logistic support. Final diagnosis of the cause of perforation is established by correlating the laparotomy findings and histopathology of margin of perforation with preoperative findings [3]. Before surgical intervention, vigorous resuscitative measures have been undertaken.

Ileal perforation is one of the critical surgical emergencies in developing country which is most prevalent in young males [4]. Delay in hospital admission is the most leading factor predisposing to complications. Presentation with features of shock is considered lethal which is also due to delay in institution of treatment [5]. Therefore, it is suggested to suspect ileal perforation in an adult male with history of fever features of peritonitis with or without pneumo-peritoneum and who is in severe toxic condition [6]. Suspected case should be promptly referred to a place where related surgical facilities are available this is the responsibility of general practitioner.

The standard source control measure for secondary peritonitis due to hollow viscus perforation is resuscitation followed by laparotomy [4]. The methods of source control for ileal perforations include primary closure, resection, and anastomosis of small gut or diverting stoma, depending on the site and number of perforations, severity of peritonitis, and general condition of the patient [5]. Thereafter, the patient is managed with antibiotics and continued postoperative care. Ileostomy serves the purpose of diversion, decompression, and exteriorization. Primary ileostomy has been found to be superior to other surgical procedures as far as the morbidity and mortality are concerned and especially so in moribund patients presenting late in course of their illness, where it proves to be a lifesaving procedure [6]. These are the types of patients that usually come to our surgical emergencies in India.

Though ileostomy is a lifesaving procedure in such cases, it may result in significant number of complications as well [7]. A small intestinal diverting stoma carries significant morbidity, mostly due to fluid/electrolyte imbalance and nutritional depletion. Peristomal skin irritation is perhaps the commonest complication of ileostomy leading to skin excoriation [8]. Other complications after ileostomy are bleeding, ischemia, obstruction, prolapse, retraction, stenosis, para-stomal herniation, fistula formation, residual abscess, wound infection, and 
incisional hernia. In addition, ileostomy is known to adversely affect the quality of life due to physical restrictions and psychological problems [9]. This present study was undertaken to see the post-operative mortality after ileal perforation.

\section{Methodology}

This cross sectional study was conducted in the Department of Surgery at Dhaka Medical College Hospital, Dhaka from September 2000 to December 2002 for period of 2 years and 3 months. All the patients presented with ileal perforation at any age with both sexes were included in this study. Patients were selected consecutively and the patients who showed ileal perforation at laparotomy were included in this study. A standard protocol was filled up for every patient. Preoperative diagnosis was based on detailed history, complete physical examinations supported by plain $\mathrm{x}$-ray abdomen in erect posture including both domes of diaphragm. After immediate resuscitation surgical treatment was undertaken as soon as possible following admission in all cases. Surgical procedures included simple repair, wedge resection and repair, resection and anastomosis, right hemi colectomy and temporary ileostomy. Histopathological examination of tissues from the margin of perforation and mesenteric lymph nodes were carried out in almost all cases. Per-operative findings and histopathological reports and laboratory investigations were correlated with preoperative findings to establish the diagnosis of ileal perforation and to elucidate an etiology. In this study, ileal perforation due to typhoid, TB, trauma, non-specific inflammation were included. Histopathological studies were carried out in the pathological laboratory of Dhaka Medical College. Hematological, bacteriological, serological tests were carried out in the laboratories of Dhaka Medical college Hospital, Dhaka. A meticulous postoperative care and follow up were done in all cases during their stay in hospital and complications were observed. Those patients who developed postoperative complications remained in the hospital as long as necessary and relevant investigations were done and proper management were undertaken. All patients received specific treatment for underlying diseases. In this study we have only considered early postoperative complications which included wound infection and dehiscence, respiratory complications, septicemia, residual abscess, paralytic Ileus, burst abdomen and faecal fistula. For the purpose detecting factors predisposing to complications we have considered relationship between postoperative complications and number of variables of which delayed presentation and association with typhoid lever have been emphasized.

\section{Result}

A total number of 100 patients were recruited for this study. Age of the patient varied from 12 years to 70 years average being 27.73 years. Third decade comprises the largest age group. Average age of male was 26.46 years and female was 31.35 years. Most of the patients who developed complications belonged to second decade. Incidence of complications was noted more in patients older 
than 40 years i.e. admitted all patients above 40 years developed complications. Out of 100 patients 74 were male and 26 were female (Table 1).

In this study 53 patients were reported complication after surgery. Out of 53 patients having postoperative complications 38 patients were survived and 15 patients were expired. Thus, 15 patients died out of 100 patients giving an overall mortality rate $15 \%$ cases. Therefore, total survivors were $85.0 \%$ cases and non-survivors $15.0 \%$ cases (Table 2).

In this study out of 15 death cases male was reported in 5 cases and the rest 10 cases were female. Majority patients were in the age group of 21 to 40 years which was 7 cases followed by less than 20 years and 41 to 60 years of age group which was 3 cases in each group. Thus the mortality rate was higher in female and older age groups (Table 3 ).

The actual cause of death could not be ascertained as the postmortem pathology could not be done. This is roughly a clinical observation from which we can assume that this type of complications is associated with increased number of mortality. In this study most of the mortality (12\%) was attributed to septicemia and mode of death was multiple organ failure. Respiratory complications caused $2 \%$ mortality one patient died of ARDS and another elderly patient with preexisting bronchial asthma developed respiratory failure and was unresponsive to treatment. One death was related to faecal fistula followed by severe fluid, electrolyte and acid base imbalance with peritonitis and gross sepsis (Table 4).

The mortality was significantly related to typhoid ulcer perforation which was $12(79.9 \%)$ cases. Intestinal TB, trauma and non-specific causes were found in 1 case in each (Table 5).

Table 1. Age and gender distribution of study population $(n=100)$.

\begin{tabular}{cccc}
\hline Age Group & Male & Female & Total \\
\hline 11 to 20 Years & 22 & 4 & $26(26.0 \%)$ \\
21 to 30 Years & 35 & 9 & $44(44.0 \%)$ \\
31 to 40 Years & 10 & 10 & $20(20.0 \%)$ \\
41 to 50 Years & 4 & 1 & $5(5.0 \%)$ \\
51 to 60 Years & 2 & 1 & $3(3.0 \%)$ \\
More than 6 Years & 1 & $26(26.0 \%)$ & $2(2.0 \%)$ \\
Total & $74(74.0 \%)$ & $31.4 \pm 10.23$ & $100(100.0 \%)$ \\
Mean \pm SD & $26.5 \pm 11.34$ & & \\
\hline
\end{tabular}

Table 2. Outcomes of postoperative complications $(n=53)$.

\begin{tabular}{cccc}
\hline Outcomes & Male & Female & Total \\
\hline Survived & $32(86.5 \%)$ & $6(37.5 \%)$ & $38(71.7 \%)$ \\
Expired & $5(13.5 \%)$ & $10(62.5 \%)$ & $15(28.3 \%)$ \\
Total & $37(100.0 \%)$ & $16(100.0 \%)$ & $53(100.0 \%)$ \\
\hline
\end{tabular}


Table 3. Age and gender distribution of expired patients $(n=15)$.

\begin{tabular}{cccc}
\hline Age Group & Male & Female & Total \\
\hline Less than 20 Years & 1 & 2 & 3 \\
21 to 40 Years & 1 & 6 & 7 \\
41 to 60 Years & 2 & 1 & 3 \\
More than 60 Years & 1 & 1 & 2 \\
Total & $\mathbf{5 ( 6 . 7 5 \% )}$ & $\mathbf{1 0 ( 3 8 . 4 6 \% )}$ & $\mathbf{1 5}(\mathbf{1 5 . 0 \% )}$ \\
\hline
\end{tabular}

Table 4. Post-operative complications in death cases among ileal perforated patients $(n=15)$.

\begin{tabular}{ccc}
\hline Postoperative complications & Frequency & Percent \\
\hline Septicemia & 12 & 12.0 \\
Respiratory complications & 2 & 2.0 \\
Faecal fistula with peritonitis & 1 & 1.0 \\
Total & 15 & 15.0 \\
\hline
\end{tabular}

Table 5. Causes of ileal perforation among death cases $(n=15)$.

\begin{tabular}{ccc}
\hline Causes & Frequency & Percent \\
\hline Typhoid & 12 & $79.9 \%$ \\
TB & 1 & $6.7 \%$ \\
Trauma & 1 & $6.7 \%$ \\
Non-specific & 1 & $6.7 \%$ \\
Total & 15 & 100.0 \\
\hline
\end{tabular}

\section{Discussion}

Despite the many improvements in surgical and anesthetic techniques in current years, respiratory complications remain a common postoperative problem [6]. However, incidence of respiratory complications is much lower in different studies. This downward trend of incidence is probably due to the overall improvement of perioperative patient management and increased awareness [7]. Respiratory complications are observed more in elderly patients in smoker, in patients with coexisting pulmonary disease and associated abdominal distension. The emergency nature of the operations without adequate preoperative evaluation and poor general condition of the patents made them especially vulnerable one factor that also predisposes to respiratory complications is repeated use of same disposable endotracheal tube without proper cleaning [8] [9].

Various types of respiratory complications encountered in this sense minor infection, atelectasis, pneumonia and so on. One patient developed acute respiratory distress syndrome (ARDS) and another elderly patient with preexisting Bronchial asthma developed respiratory failure. Their condition is so grave despite necessary management in intensive care unit they expired. Adequate nursing, early ambulation and chest physiotherapy are the possible way to prevent postoperative complications [6]. Administration of low doses of analgesics at frequent intervals pro- 
motes improved respiration at postoperative period by controlling pain without over sedation [3].

Age of the patient varied from 12 years to 70 years average being 27.73 years. Third decade comprises the largest age group. Average age of male was 26.46 years and female was 31.35 years. Most of the patients who developed complications belonged to second decade. Incidence of complications was noted more in patients older than 40 years i.e. admitted all patients above 40 years developed complications. Out of 100 patients 74 were male and 26 were female. Essentials of treatment of faecal fistula include fluid and electrolyte resuscitation, control of sepsis, protection of skin, and maintenance of adequate nutrition and surgical intervention where it is indicated [10]. In this study incidence of overall complication was much lower than those of other studies except septicemia. Among the above complications 12 patients from septicemia, 2 patents from respiratory complications and one patient from faecalis fistula lead to fatality. The total Mortality rate was $15 \%$ which was also lower than that of other studies. So early diagnosis and treatment with special care to prevent complications are necessary if the mortality and morbidity statistics are to be improved.

Though this study has been conducted a long year back, the results reflect the serious scenario. The developing country like Bangladesh is facing a huge burden of surgical interventions. In this regards this study still has a good impact on the people of this country. Out of 53 complicated patients 15 patients has been died giving an overall mortality rate 15.0\%; 38 patients survived and had long hospital stay. The postoperative complications which related with mortality are septicemia (12.0\%), pulmonary complications $(2.0 \%)$ and faecal fistula (1.0\%). Factors predisposing to complications are identified female sex, older age, poor socio-economic condition with poor nutritional status, delayed presentation, prolonged fever, shock at admission, associated illness like jaundice, anaemia, preexisting lung diseases, generalized abdominal distension, gross peritoneal contamination with purulent peritoneal fluid, multiple perforations and operative procedure [11]. Mortality, morbidity and duration of hospital stay were observed to be lower in this study as compared to other studies [12] [13]. This improved picture is attributed to aggressive preoperative resuscitation, use of newer more efficient antibiotics, and early precise surgery along with adequate peritoneal toileting, meticulous postoperative management and prompt dealing of complications [5].

There are some limitations of this study. The sample size is small. Furthermore this is a single centre study. In addition this is a study of few years back.

\section{Conclusion}

In conclusion, most of the mortalities are attributed to septicemia and mode of death is multiple organ failure. Respiratory complications are also recorded which is diagnosed as acute respiratory distress s syndrome (ARDS). Death of ileal perforation patients is also due to fecal fistula followed by severe fluid, electrolyte and acid-base imbalance with peritonitis and gross sepsis. Further multi-centers large scale study should be carried out countrywide to get more accurate pictures. 


\section{Conflicts of Interest}

The authors declare no conflicts of interest regarding the publication of this paper.

\section{References}

[1] Bali, R.S., Verma, S., Agarwal, P.N., Singh, R. and Talwar, N. (2014) Perforation Peritonitis and the Developing World. ISRN Surgery, 2014, Article ID: 105492. https://doi.org/10.1155/2014/105492

[2] Yadav, D. and Garg, P.K. (2013) Spectrum of Perforation Peritonitis in Delhi: 77 Cases Experience. Indian Journal of Surgery, 75, 133-137. https://doi.org/10.1007/s12262-012-0609-2

[3] Gupta, S. and Kaushik, R. (2006) Peritonitis-The Eastern Experience. World Journal of Emergency Surgery, 1, Article No. 13. https://doi.org/10.1186/1749-7922-1-13

[4] Afridi, S.P., Malik, F., Ur-Rahman, S., Shamim, S. and Samo, K.A. (2008) Spectrum of Perforation Peritonitis in Pakistan: 300 Cases Eastern Experience. World Journal of Emergency Surgery, 3, Article No. 31. https://doi.org/10.1186/1749-7922-3-31

[5] Jhobta, R.S., Attri, A.K., Kaushik, R., Sharma, R. and Jhobta, A. (2006) Spectrum of Perforation Peritonitis in India-Review of 504 Consecutive Cases. World Journal of Emergency Surgery, 1, Article No. 26. https://doi.org/10.1186/1749-7922-1-26

[6] Chakma, S.M., Singh, R.L., Parmekar, M.V., Singh, K.G., Kapa, B., Sharatchandra, K.H., et al. (2013) Spectrum of Perforation Peritonitis. Journal of Clinical and Diagnostic Research, 7, 2518-2520. https://doi.org/10.7860/JCDR/2013/5768.3596

[7] Gupta, S.K., Gupta, R., Singh, G. and Gupta, S. (2010) Perforation Peritonitis: A Two Year Experience. JK Science, 123, 141-144.

[8] Asif, A., Byers, P., Vieira, C.F., Merrill, D., Gadalean, F., Bourgoignie, J.J., et al. (2003) Peritoneoscopic Placement of Peritoneal Dialysis Catheter and Bowel Perforation: Experience of an Interventional Nephrology Program. American Journald of Kidney Diseases, 42, 1270-1274. https://doi.org/10.1053/j.ajkd.2003.08.029

[9] Ekenze, S.O. and Okoro, P.E. (2008) Typhoid Ileal Perforation: Analysis of Morbidity and Mortalityin 89 Children. Nigerian Journal of Clinical Practice, 11, 59-63.

[10] Verma, H., Pandey, S., Sheoran, K.D. and Marwah, S. (2015) Surgical Audit of Patients with Ileal Perforations Requiring Ileostomy in a Tertiary Care Hospital in India. Surgery Research and Practice, 2015, Article ID: 351548. https://doi.org/10.1155/2015/351548

[11] Nuhu, A., Dahwa, S. and Hamza, A. (2010) Operative Management of Typhoid Ileal Perforation in Children. African Journal of Paediatric Surgery, 7, 9-13. https://doi.org/10.4103/0189-6725.59351

[12] Nema, A.A. and Darshan, J.R. (2018) A Study of Surgical Approach to Typhoid Ileal Perforation at a Tertiary Care Hospital of South Gujarat, India. International Surgery Journal, 5, 1758-1762. https://doi.org/10.18203/2349-2902.isj20181458

[13] Mishra, M., Singh, P. and Tripathi, A. (2018) Typhoid Ileal Perforation: Comparative Study of Ileostomy versus Primary Ileal Repair and Associated Morbidity and Mortality. International Surgery Journal, 5, 3129-3133. https://doi.org/10.18203/2349-2902.isj20183735 